Chemcomm

Chemical Communications

rsc.li/chemcomm
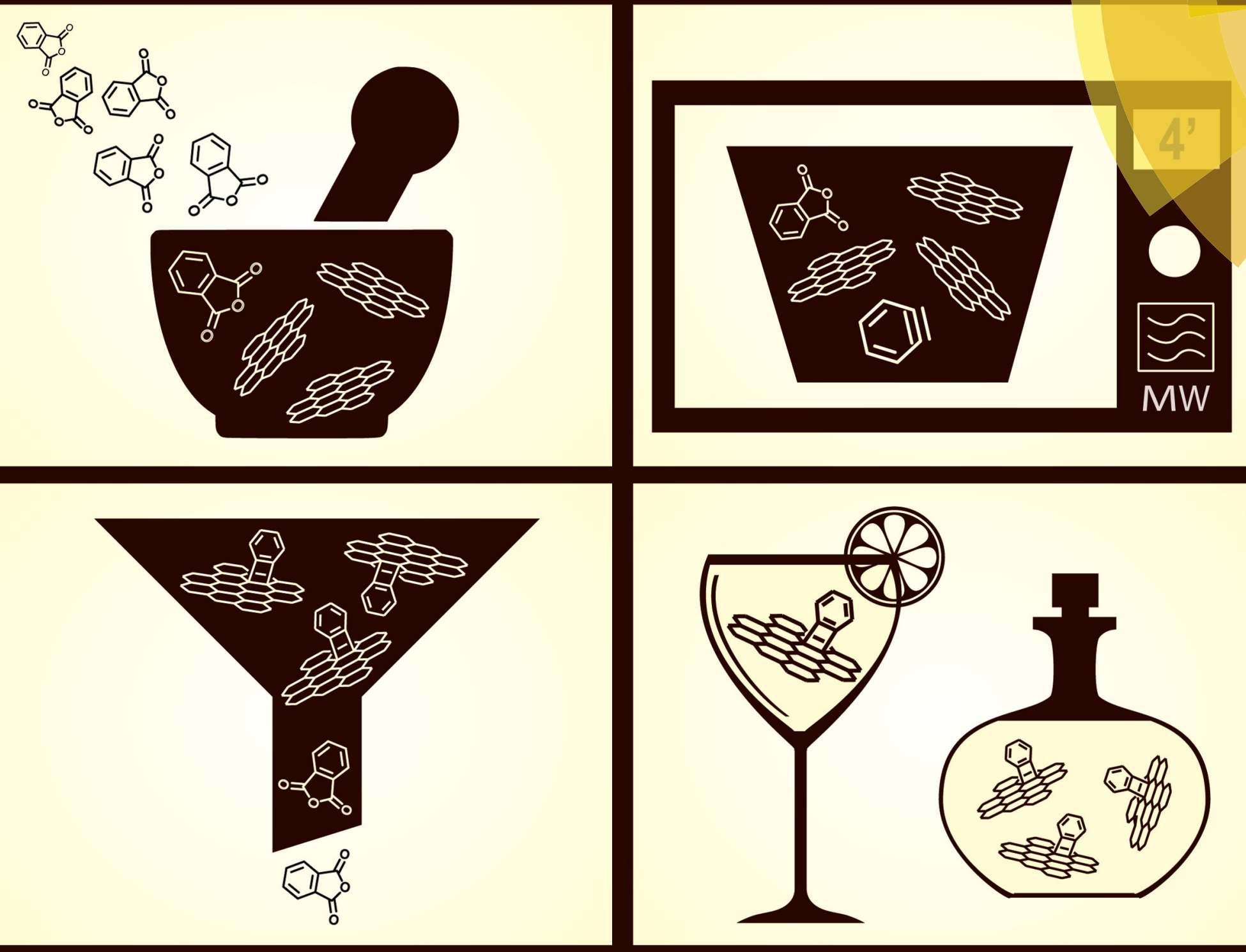

ISSN 1359-7345 
Check for updates

Cite this: Chem. Commun., 2018, 54, 2086

Received 10th November 2017, Accepted 4th January 2018

DOI: $10.1039 / \mathrm{c7cc08676h}$

rsc.li/chemcomm

\section{Microwave-induced covalent functionalization of few-layer graphene with arynes under solvent-free conditions $\uparrow$}

\author{
M. V. Sulleiro, ${ }^{a}$ S. Quiroga, ${ }^{b}$ D. Peña, ${ }^{b}$ D. Pérez, ${ }^{b}{ }^{b}$ E. Guitián, ${ }^{b}$ A. Criado ID *c and \\ M. Prato iD *acd
}

induced decomposition of $o$-(trimethylsilyl)aryl triflates. ${ }^{12}$ However, in many cases the synthesis of these aryne precursors is not easily performed and their decomposition usually needs fluoride ions that can interfere in the reaction and alter the functionalized carbonbased material. ${ }^{13}$ Thus, establishment of fast and scalable protocols for GBM functionalization with accessible reagents is still needed.

Microwave-assisted (MW) chemistry is an important tool in the field of carbon-based nanomaterials. ${ }^{7 a, b}$ In particular, GBMs and in general carbon-based materials are extensively used as MW matrices because of their capacity to absorb MW with high efficiency, rapidly generating high surface temperatures. ${ }^{14}$ In addition, different methods for the preparation of graphene derivatives have been recently reported by using MW irradiation. ${ }^{15}$

In this work we report a new, facile, rapid and nonconventional approach to efficiently and mildly functionalize GBMs under MW irradiation and solvent-free conditions. We focused on underexplored cycloaddition reactions of arynes with exfoliated few-layer graphene (FLG). ${ }^{12 a, b}$ In particular, different arynes were generated by the thermal decomposition of the corresponding arylene anhydrides at high temperatures. Due to the good MW absorption, GBMs played two roles in the reaction process: as a reagent and, at the same time, as a MW absorbing matrix which allows high temperatures to be reached in short times under solvent-free conditions.

The pristine graphene material used in this work was obtained through exfoliation from graphite following Coleman's protocol. ${ }^{16}$ Raman spectroscopy showed that the produced graphene consisted of a few layers. ${ }^{17}$ The $2 \mathrm{D}$ band of the pristine graphene materials can be deconvoluted into four Lorentzian-shaped peaks (Fig. S9, ESI $\dagger$ ), which is a characteristic feature of few-layer (less than five) graphene as previously reported. In addition, the spectrum is also quite different from bulk graphite (Fig. S11, ESI $\dagger$ ).

We initially investigated a controllable chemical functionalization of FLG with benzyne generated by the thermal decomposition of the commercially available phthalic anhydride (1). ${ }^{18}$ The benzyne precursor 1 and the FLG were thoroughly mixed in a mortar, then the homogeneous mixture was rapidly heated up under MW irradiation and solvent-free conditions (Fig. 1). 


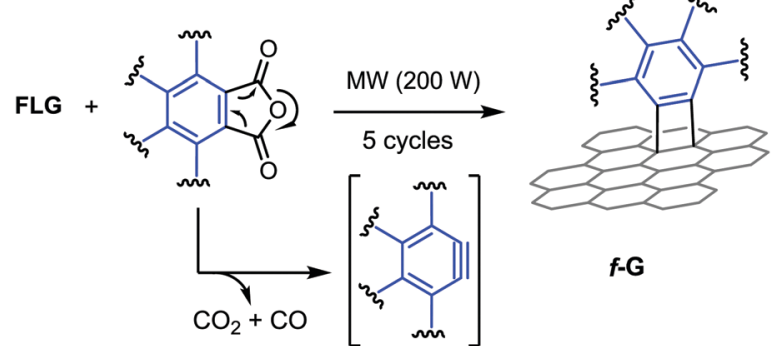

Fig. 1 Functionalization of FLG with arynes by the thermal decomposition of arylene anhydrides.

After considering different reaction parameters such as number of equivalents, temperature, reaction time, and power irradiation, we concluded that the irradiation power is the key to modulate the functionalization degree. Thus, different homogenous powder mixtures of FLG and $\mathbf{1}$ were carried out at different cycles of MW irradiation. In each cycle, 200 watts of MW power were applied until reaching $250{ }^{\circ} \mathrm{C}$, in about 5 seconds (Fig. S12, ESI $\dagger$ ).

The reaction evolution on the microwave reactor was followed by thermogravimetric analysis (TGA) and Raman spectroscopy, clearly confirming the chemical modification of graphene with benzyne (Fig. 2). TGA allows the estimation of the functionalization degree (FD), defined as the number of micromole of functional groups per gram of sample (eqn (S1), ESI $\dagger$ ). The TGA results show significant variations in the FD depending on the number of cycles, while a slight increase in the $I_{\mathrm{D}} / I_{\mathrm{G}}$ ratio using Raman was observed. Both $I_{\mathrm{D}} / I_{\mathrm{G}}$ ratios and FDs are in accordance up to 5 cycles of MW irradiation, where the corresponding functionalized graphene (f-G) presented a FD of $122 \mu \mathrm{mol} \mathrm{g}{ }^{-1}$ with a $I_{\mathrm{D}} / I_{\mathrm{G}}$ ratio of 0.29 (Table S1, $\mathrm{ESI} \dagger$ ). However, after 5 cycles, the functionalization degree observed using TGA decreases while the defects still mildly increase, observed using Raman. It is worth mentioning that this chemical process is performed at high temperatures. Probably, the functional groups are partially cleaved probably through the retro-cycloaddition of benzyne under the reaction conditions. ${ }^{10,19}$ In fact, theoretical studies support this possibility as they have reported energy barriers of $1.9 \mathrm{eV}$ for the retro-cycloaddition reaction of the corresponding adduct of benzyne with graphene, which can be overcome at moderately high temperatures. ${ }^{19 a}$ Accordingly, functionalized graphene materials with more than 5 cycles showed smaller FD with a high $I_{\mathrm{D}} / I_{\mathrm{G}}$ ratio. In addition, defects can be introduced in

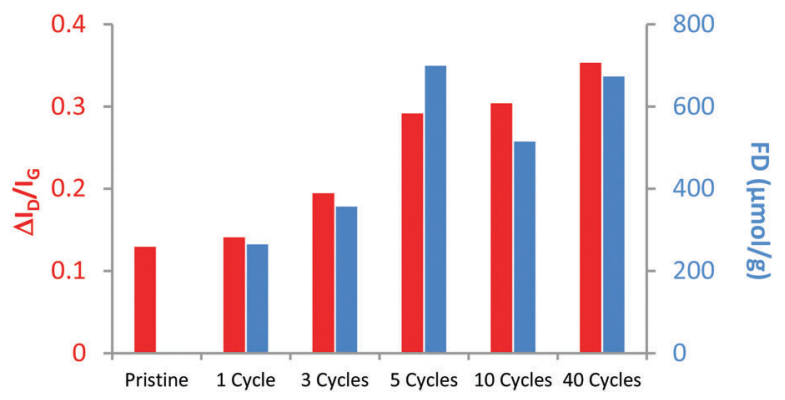

Fig. $2 I_{D} / I_{G}$ ratio (red) and $F D$ (blue) for $f-G(7)$ at different cycles. the graphene lattice after a large number of cycles, independently of the organic functionalization with benzyne. This last assessment is supported by TGA profiles of functionalized graphene with arynes, where a thermal decomposition is observed after around $200{ }^{\circ} \mathrm{C}$ (red square in Fig. S13, ESI $\dagger$ ). Therefore, the 5 cycle modification was established as the standard method because it generates an adequate FD in line with the $I_{\mathrm{D}} / I_{\mathrm{G}}$ ratio. The morphology of the functionalized materials was studied using transmission electron microscopy (TEM, Fig. S15-S18, ESI $\dagger$ ). The graphene derivative f-G(7) (Fig. 3) keeps a similar structure and dimensions of the pristine material after the described treatment at high temperatures. Besides, it was not possible to observe organic layers on the graphene surface from these images, thus supporting our previous claim about the molecular functionalization.

To confirm the generation of aryne under the established reaction conditions, the "capture" of the generated benzyne was tried by adding anthracene as a thermally stable diene into the solid mixture, because it can yield the easily characterized aromatic compound triptycene (15), derived from a $[4+2]$ cycloaddition (Fig. 4). The NMR analysis of the reaction mixture led to 15 as the major product in approximately $33 \%$ conversion, and 60 and $27 \%$ of recovered 1 and 13, respectively (Fig. S8, ESI $\dagger$ ). This result successfully proves the generation of $\mathbf{1 4}$ from $\mathbf{1}$ by thermal decomposition under the described conditions.

To date, aryne cycloaddition reactions with diverse carbon nanomaterials have been reported. For example, benzyne cycloaddition with fullerene $\mathrm{C}_{60}$ leads to $[2+2]$ adducts. ${ }^{20}$ Nevertheless, for reactions performed on carbon nanotubes, ${ }^{10}$ carbon nanohorns, ${ }^{11}$ and graphene derivatives, ${ }^{12}$ there is no experimental evidence as yet about the exact structure of the adducts obtained. Several theoretical studies suggest that the benzyne reaction with carbon nanotubes presents periselectivity (i.e., either [4+2] or $[2+2])$ depending on different parameters, such as the type of carbon nanotube (i.e., number of walls, chirality, etc.) and its diameter. ${ }^{10 a, 21}$ In the case of graphene, theoretical studies argued that the reaction with benzyne on a graphene fragment of $4 \times 4$, $6 \times 6$ and $8 \times 8$ unit cells is more energetically favorable through $[2+2]$ cycloaddition, but both can occur. ${ }^{19 b, 21 c}$ Other theoretical calculations suggest that cycloaddition reactions occur at edges and defected areas. ${ }^{19 a, 22}$ However, cycloaddition reactions have been also reported on defect-free epitaxial graphene. ${ }^{8 b}$

The described graphene functionalization was extended to structurally more complex arynes (Fig. 3) via thermal decomposition of the corresponding anhydrides (2-6) under the standard conditions described for phthalic anhydride. In particular, arylene groups with heteroatoms, such as a fluorine-substituted phenylene (f-G(8)), or aromatic moieties with a large number of fused benzene rings (f-G(9-12)) have been covalently linked to the graphene structure. In the latter case, aromatic groups were introduced with the same number of benzene rings but with different topologies (f-G(10-11)). The new modified graphene derivatives were characterized using Raman spectroscopy, TGA, TEM, as well as XPS techniques, showing consistent functionalization yields in all cases (Table S2, ESI $\dagger$ ).

In addition, X-ray photoelectron spectroscopy (XPS) was used to provide evidence for the formation of functionalized 
<smiles>[R]c1c([R])c([R])c2c(c1[R])C(=O)OC2=O</smiles>

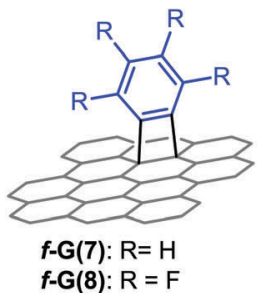<smiles>O=C1OC(=O)c2cc3ccccc3cc21</smiles>

3

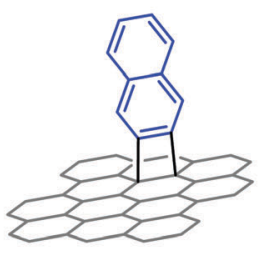

$f-G(9)$<smiles>O=C1OC(=O)c2cc3cc4ccccc4cc3cc21</smiles>

4

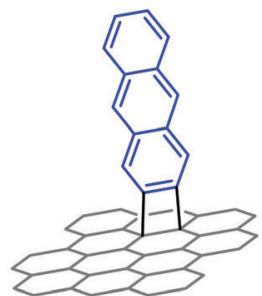

$f-G(10)$
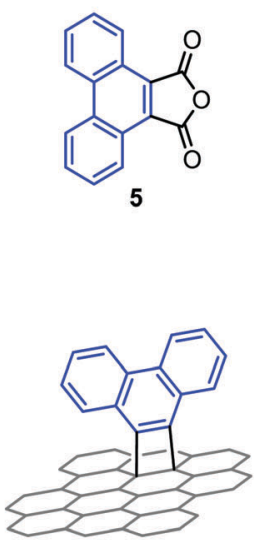

$f-G(11)$
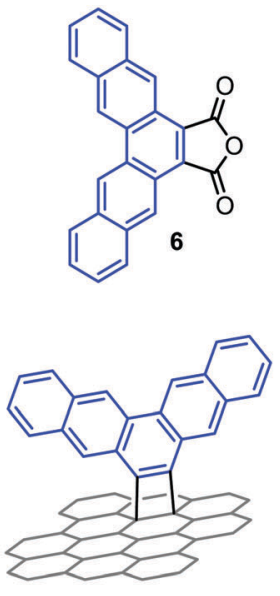

$f-G(12)$

Fig. 3 Chemical representation of the arylene anhydrides 1-6 to obtain $f-G(\mathbf{7 - 1 2})$, under MW irradiation and solvent-free conditions

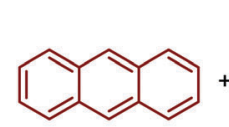

13<smiles>O=C1OC(=O)c2ccccc21</smiles>

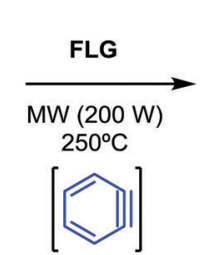

14

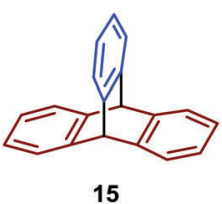

15
Fig. 4 Trapping of benzyne (14) generated upon MW irradiation of 1 under solvent-free conditions with FLG as the MW absorbing matrix.

graphene derivatives by identifying and quantifying the functional groups anchored to the surface. The binding energies and peak assignments for different cores are summarized in Tables S3 and $\mathrm{S} 4$ (ESI $\dagger$ ). In particular, fluorine atoms from the aryne precursor 2 provided a diagnostic signal proving the functionalization of FLG. The atomic percentages of $\mathrm{C}, \mathrm{O}$ and $\mathrm{F}$ atoms for the FLG, $\mathrm{f}-\mathrm{G}(\mathbf{8})$, a FLG treated under optimized reaction conditions and a control sample graphene material in the absence of any aryne precursor (cs-G) are summarized in Table 1.

$\mathrm{f}-\mathrm{G}(\mathbf{8})$ has $17.5 \%$ of $\mathrm{F}$ atoms due to the introduced tetrafluorophenylene moieties. The presence of oxygen in the modified graphene derivative is probably due to oxygenated groups, generated during the exfoliation and the irradiation process (see the compositions of FLG and cs-G, respectively). The deconvolution of C1s and F1s core levels of f-G(8) (Fig. 4) showed peaks at 286.11 and $687.15 \mathrm{eV}$, respectively, corresponding to the $\mathrm{C}-\mathrm{F}$ bonds. ${ }^{23}$ The presence of fluorine and the energy of these peaks suggest the presence of tetrafluorophenylene moieties in the graphene structure, confirming the chemical modification.

The functionalization of graphene is expected to improve the dispersibility of the graphene material in organic solvents, because the introduced functional groups can preclude re-aggregation and subsequent precipitation. ${ }^{12 b, 24}$ In order to explore the dispersion stability of the functionalized few-layer graphene materials, different solvents were tested. The f-Gs exhibited significantly improved solubility in ethanol and DMF, after sonication for
Table 1 Atomic ratios of FLG, $f-G(8)$ and $c s-G$ determined from the XPS survey spectra

\begin{tabular}{lllc}
\hline GBM & Core & BE $(\mathrm{eV})$ & Atomic (\%) \\
\hline FLG & C1s & 284.40 & 98.1 \\
& O1s & 531.6 & 1.9 \\
f-G(8) & C1s & 284.31 & 79.7 \\
& O1s & 532.76 & 2.8 \\
& F1s & 687.01 & 17.5 \\
cs-G $^{a}$ & C1s & 284.37 & \\
& O1s & 531.46 & 63.8
\end{tabular}

${ }^{a}$ FLG treated with 5 cycles of $200 \mathrm{~W}$ MW irradiation for $5 \mathrm{~s}$ each cycle in the absence of aryne precursors.

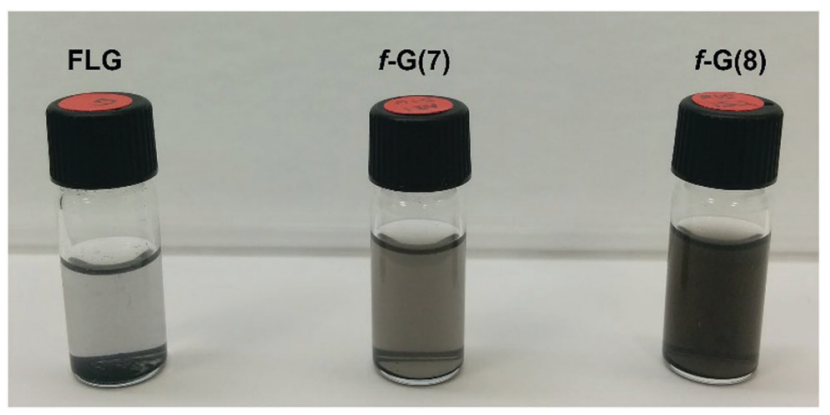

Fig. 5 Suspensions of $F L G, f-G(7)$ and $f-G(8)$ (from left to right) in $E t O H$ $\left(0.2 \mathrm{mg} \mathrm{mL}^{-1}\right)$ after 2 weeks.

5 min (Fig. S19, ESI $\dagger$ ). The f-Gs showed an improved dispersibility in ethanol after 15 days (Fig. 5), especially f-G(8).

In conclusion, we have developed a novel, fast, scalable and simple entry to covalently modified graphene. This approach shows further advantages than classical functionalization reactions, since it allows avoiding unstable graphene suspensions at low concentrations by the introduction of moieties of different nature. It is worthy to note that such moieties must be thermally stable due to the necessary high temperatures employed in the method. 
The addition of a variety of functional groups will be considered in a further work to study their influence in exfoliation and suspension stability. Therefore, the described solvent-free conditions pave the way for green protocols, large-scale functionalization processes of graphene materials and new methods to modulate electronic properties of graphene derivatives. Besides, to the best of our knowledge, it is the first chemical modification where GBMs behave like unique MW absorbing matrixes and reagents at the same time in covalent modification.

M. P. is the recipient of the AXA Chair (2016-2023). This work was supported by the EU H2020-Adhoc-2014-20 Graphene Core1 (no. 696656).

\section{Conflicts of interest}

There are no conflicts to declare.

\section{Notes and references}

1 K. S. Novoselov, A. K. Geim, S. V. Morozov, D. Jiang, Y. Zhang, S. V. Dubonos, I. V. Grigorieva and A. A. Firsov, Science, 2004, 306, 666-669.

2 P. Wick, A. E. Louw-Gaume, M. Kucki, H. F. Krug, K. Kostarelos, B. Fadeel, K. A. Dawson, A. Salvati, E. Vázquez, L. Ballerini, M. Tretiach, F. Benfenati, E. Flahaut, L. Gauthier, M. Prato and A. Bianco, Angew. Chem., Int. Ed., 2014, 53, 2-7.

3 (a) A. C. Ferrari, F. Bonaccorso, V. Fal'ko and K. S. Novoselov, et al., Nanoscale, 2015, 7, 4598-4810; (b) A. K. Geim, Science, 2009, 324, 1530-1534.

4 (a) A. Criado, M. Melchionna, S. Marchesan and M. Prato, Angew. Chem., Int. Ed., 2015, 54, 10734-10750; (b) S. Eigler and A. Hirsch, Angew. Chem., Int. Ed., 2014, 53, 7720-7738; (c) L. Rodríguez-Pérez, M. Á. Herranz and N. Martín, Chem. Commun., 2013, 49, 3721-3735.

5 (a) M. Quintana, K. Spyrou, M. Grzelczak, W. R. Browne, P. Rudolf and M. Prato, ACS Nano, 2010, 4, 3527-3533; (b) M. Quintana, A. Montellano, A. E. del Rio Castillo, G. Van Tendeloo, C. Bittencourt and M. Prato, Chem. Commun., 2011, 47, 9330-9332.

6 (a) Y. Hernandez, V. Nicolosi, M. Lotya, F. M. Blighe, Z. Sun, S. De, I. T. McGovern, B. Holland, M. Byrne, Y. K. Gun'Ko, J. J. Boland, P. Niraj, G. Duesberg, S. Krishnamurthy, R. Goodhue, J. Hutchison, V. Scardaci, A. C. Ferrari and J. N. Coleman, Nat. Nanotechnol., 2008, 3, 563-568; (b) J. N. Coleman, Acc. Chem. Res., 2013, 46, 14-22.

7 (a) E. Vázquez, F. Giacalone and M. Prato, Chem. Soc. Rev., 2014, 43, 58-69; (b) E. Vázquez and M. Prato, ACS Nano, 2009, 3, 3819-3824; (c) H. J. Salavagione, J. Mater. Chem. A, 2014, 2, 7138-7146.

8 (a) K. Kostarelos, L. Lacerda, G. Pastorin, W. Wu, S. Wieckowski, J. Luangsivilay, S. Godefroy, D. Pantarotto, J.-P. Briand, S. Muller, M. Prato and A. Bianco, Nat. Nanotechnol., 2007, 2, 108-113; (b) L. Daukiya, C. Mattioli, D. Aubel, S. Hajjar-Garreau, F. Vonau, E. Denys, G. Reiter, J. Fransson, E. Perrin, M.-L. Bocquet, C. Bena, A. Gourdon and L. Simon, ACS Nano, 2017, 11, 627-634; (c) M. Barrejón, M. J. Gómez-Escalonilla, J. L. G. Fierro, P. Prieto, J. R. Carrillo, A. Rodriguez, G. Abellán, M. C. LópezEscalante, M. Gabás, J. T. López Navarrete and F. Langa, Phys. Chem. Chem. Phys., 2016, 18, 29582-29590.
9 J.-M. Seo and J.-B. Baeka, Chem. Commun., 2014, 50, 14651-14653. 10 (a) A. Criado, M. Vizuete, M. J. Gómez-Escalonilla, S. García-Rodriguez, J. L. G. Fierro, A. Cobas, D. Peña, E. Guitián and F. Langa, Carbon, 2013, 63, 140-148; (b) A. Criado, M. J. Gómez-Escalonilla, J. L. G. Fierro, A. Urbina, D. Peña, E. Guitián and F. Langa, Chem. Commun., 2010, 46, 7028-7030.

11 D. Chronopoulos, N. Karousis, T. Ichihashi, M. Yudasaka, S. Iijima and N. Tagmatarchis, Nanoscale, 2013, 5, 6388-6394.

12 (a) D. García, L. Rodríguez-Pérez, M. A. Herranz, D. Peña, E. Guitián, S. Bailey, Q. Al-Galiby, M. Noori, C. J. Lambert, D. Pérez and N. Martín, Chem. Commun., 2016, 52, 6677-6680; (b) X. Zhong, J. Jin, S. Li, Z. Niu, W. Hu, R. Li and J. Ma, Chem. Commun., 2010, 46, 7340-7342; (c) I. V. Magedov, L. V. Frolova, M. Ovezmyradov, D. Bethke, E. A. Shaner and N. G. Kalugin, Carbon, 2013, 54, 192-200.

13 C. Romero, D. Peña, D. Pérez and E. Guitián, Chem. - Eur. J., 2006, $12,5677-5684$.

14 (a) H. Y. Cho, A. Ajaz, D. Himali, P. A. Waske and R. P. Johnson, J. Org. Chem., 2009, 74, 4137-4142; (b) N. D. Kim, A. Metzger, V. Hejazi, Y. Li, A. Kovalchuk, S. K. Lee, R. Ye, J. A. Mann, C. Kittrell, R. Shahsavari and J. M. Tour, ACS Appl. Mater. Interfaces, 2016, 8, 12985-12991; (c) H. Naeimi and M. Golestanzadeh, New J. Chem., 2015, 39, 2697-2710.

15 (a) M. Matsumoto, Y. Saito, C. Park, T. Fukushima and T. Aida, Nat. Chem., 2015, 7, 730-736; (b) D. Ghosh, G. Halder, A. Sahasrabudhe and S. Bhattacharyya, Nanoscale, 2016, 8, 10632-10641; (c) H. Luo, R. Gong, X. Wang, K. Song, C. Zhu and L. Wang, New J. Chem., 2016, 40, 6238-6243; (d) A. Akın and N. Ișılklan, Int. J. Biol. Macromol., 2016, 82, 530-540; (e) K. Cao, Y. Tian, Y. Zhang, X. Yang, C. Bai, Y. Luo, X. Zhao, L. Ma and S. Li, Nanoscale, 2014, 6, 13518-13526; $(f)$ Y. M. Shulga, S. A. Baskakov, E. I. Knerelman, G. I. Davidova, E. R. Badamshina, N. Y. Shulga, E. A. Skryleva, A. L. Agapov, D. N. Voylov, A. P. Sokolov and V. M. Martynenko, RSC Adv., 2014, 4, 587-592.

16 S. Barwich, U. Khan and J. N. Coleman, J. Phys. Chem. C, 2013, 117, 19212-19218.

17 (a) A. C. Ferrari, J. C. Meyer, V. Scardaci, C. Casiraghi, M. Lazzeri, F. Mauri, S. Piscanec, D. Jiang, K. S. Novoselov, S. Roth and A. K. Geim, Phys. Rev. Lett., 2006, 97, 187401; (b) L. M. Malard, M. A. Pimenta, G. Dresselhaus and M. S. Dresselhaus, Phys. Rep., 2009, 473, 51-87.

18 E. K. Fields and S. Meyerson, J. Am. Chem. Soc., 1966, 31, 3307.

19 (a) J. Zhao, H. Wang, B. Gao, X. Wang, Q. Cai and X. Wang, J. Mol. Model., 2012, 18, 2861-2868; (b) P. A. Denis and F. Iribarne, J. Mater. Chem., 2012, 22, 5470-5477; (c) S. Sarkar, E. Bekyarova, S. Niyogi and R. C. Haddon, J. Am. Chem. Soc., 2011, 133, 3324-3327.

20 S. H. Hoke, J. Molstad, D. Dilettato, M. J. Jay, D. Carlson, B. Kahr and R. G. Cooks, J. Org. Chem., 1992, 57, 5069-5071.

21 (a) T. Yang, X. Zhao and S. Nagase, Org. Lett., 2013, 15, 5960-5963; (b) J. P. Martínez, F. Langa, F. Matthias Bickelhaupt, S. Osuna and M. Solá, J. Phys. Chem. C, 2016, 120, 1716-1726; (c) M. Hammouri, S. K. Jha and I. Vasiliev, J. Phys. Chem. C, 2015, 119, 18719-18728.

22 (a) Y. Cao, S. Osuna, Y. Liang, R. C. Haddon and K. N. Houk, J. Am. Chem. Soc., 2013, 135, 17643-17649; (b) P. A. Denis, Chem. - Eur. J., 2013, 19, 15719-15725.

23 J. F. Moulder, W. F. Stickle, P. E. Sobol and K. D. Bomben, Handbook of X-Ray Photoelectron Spectroscopy, ed. J. Chastain and R. King Jr., Physical Electronics, Inc., 3rd edn, 1995.

24 (a) A. Ciesielski and P. Samorì, Chem. Soc. Rev., 2014, 43, 381-398; (b) J. M. Englert, C. Dotzer, G. Yang, M. Schmid, C. Papp, E. Spiecker, F. Hauke, A. Hirsch, J. M. Gottfried and H. Steinru, Nat. Chem., 2011, 3, 279-286. 